\title{
OBTENÇÃO E CARACTERIZAÇÃO DE NANOESTRUTURAS DE CARBONATO DE CÁLCIO.
}

\author{
B. E. R. PAZ ${ }^{1}$, C. C. V. VELLOSO ${ }^{2}$, E. N. O. JÚNIOR², J. S. R. COIMBRA³ e I. J. B. \\ SANTOS $^{2}$ \\ ${ }^{1}$ Universidade Federal de São João del-Rei, Departamento de Engenharia Química \\ ${ }^{2}$ Universidade Federal de São João del-Rei, Departamento de Engenharia de Bioprocessos \\ ${ }^{3}$ Universidade Federal de Viçosa, Departamento de Tecnologias em Alimentos
}

\section{E-mail para contato: igorboggione@ufsj.edu.br}

\begin{abstract}
RESUMO - Nanoestruturas de carbonato de cálcio possuem muitas aplicações na indústria, como aditivos nas indústrias de plástico e papel e na liberação controlada de moléculas bioativas. Nesse trabalho, nanopartículas de carbonato de cálcio foram obtidas a partir da adição controlada de solução de nitrato de cálcio a uma solução de carbonato de sódio, sob agitação constante. Foi estudado o efeito da temperatura, proporção entre as soluções para síntese de nanopartícula e a temperatura no tamanho de nanopartículas. As melhores condições para obtenção da nanoestrutura foram temperatura de $17,5{ }^{\circ} \mathrm{C}$, proporção $1: 1$ e tempo de gotejamento de 60 minutos. A partir da análise de superfícies de resposta geradas a partir da metodologia Box-Behnken, concluiu-se que quanto menor o tempo de gotejamento, menor o tamanho de partícula e que a proporção não possui impacto significativo na resposta. A partir da análise de variância (ANOVA) constatou-se que a relação da variável proporção é em regressão linear e da variável temperatura é em regressão quadrática.
\end{abstract}

\section{INTRODUÇÃO}

A nanotecnologia é um campo da tecnologia responsável pela caracterização, fabricação, manipulação e aplicação de materiais com uma ou mais dimensões em tamanho nanométrico $(1 \mathrm{~nm}$ a $300 \mathrm{~nm})$, a fim de se obter processos e produtos com melhor desempenho e/ou novas propriedades (ASSIS et al., 2014). Os nanomateriais possuem potencial impacto nos mais variados setores industriais, influenciando o desenvolvimento tecnológico e econômico do país. Isso se deve ao fato de que os produtos nanoestruturados possuem propriedades mecânicas, ópticas, magnéticas ou químicas distintas de partículas e superfícies macroscópicas. Dessa maneira, a nanotecnologia tem atraído considerável atenção nos últimos anos por parte de governos, indústrias e centros de pesquisas (BOUWMEESTER et al., 2009).

O carbonato de cálcio é um biomaterial inorgânico de ocorrência natural (MANJUSHA et al., 2014). As nanoestruturas de carbonato de cálcio são utilizadas como aditivos nas indústrias de papel e de plástico, proporcionando propriedades novas como o aumento da rigidez (BABOU-KAMMOE et. al., 2012). Além disso, essas nanopartículas também são utilizadas em estudos de liberação controlada de moléculas bioativas (HARUTA et al., 2003). 
Existem relatos na literatura sobre a atividade antimicrobiana das partículas de carbonato de cálcio em sua forma nanoestruturada (JEONG et al., 2007). Portanto, nanoestruturas de carbonato de cálcio possuem diversas aplicações e a sua obtenção é de grande interesse industrial, além de ser uma área com grande potencial para pesquisas.

Tecnologias para produzir nanopartículas de carbonato de cálcio incluem precipitação ou carbonatação úmida, utilização de membranas de emulsão e novas tecnologias como a precipitação reativa de alta gravidade. O método de precipitação é relativamente simples e já foi demonstrado ser possível sintetizar partículas superfinas usando esse método (BABOUKAMMOE et. al., 2012). Nesse trabalho, o método utilizado para a síntese de nanoestruturas de carbonato de cálcio é a adição controlada, sob agitação, de solução aquosa de nitrato de cálcio a uma solução aquosa composta de carbonato de sódio, hidróxido de sódio e nitrato de sódio. Para posterior otimização do processo, os efeitos das variáveis que influenciam a síntese de nanoestrutura de carbonato de cálcio foram analisados.

\section{METODOLOGIA}

Hidróxido de sódio e carbonato de sódio foram obtidos do laboratório ProLab (São Paulo/SP, Brasil), nitrato de sódio e nitrato de cálcio do Labsynth (Diadema/SP, Brasil). Água destilada proveniente da osmose Hidrotek (Santa Iria de Azóia, Portugal) foi utilizada em todos os experimentos. Os demais reagentes foram utilizados sem purificação adicional e foram de qualidade analítica.

\subsection{Obtenção de nanoestruturas de carbonato de cálcio}

Nanopartícula de carbonato de cálcio foi obtida de acordo com BABOU-KAMMOE et al. (2012) com modificações. Uma solução de $0,1 \mathrm{~mol}$. $\mathrm{L}^{-1}$ de carbonato de sódio, $0,2 \mathrm{~mol}$. $\mathrm{L}^{-1}$ de hidróxido de sódio e 0,18 mol. $\mathrm{L}^{-1}$ nitrato de sódio foram preparadas e nesta solução foi adicionado gota a gota uma solução de $0,1 \mathrm{M}$ de nitrato de cálcio sob agitação constante, a $25^{\circ} \mathrm{C}$ e a $50^{\circ} \mathrm{C}$ por 60 min.. Após esse tempo, a solução foi centrifugada (Eppendorf Centrifuge 5804;5804 R- BioResearch do Brasil- Made in Germany) a 3500 rpm durante 10 min e o pellet obtido foi seco em estufa (Estufa Bacteriológica, Solab Equipamentos para Laboratórios LTDA- EPP, SL 101/81, Piracicaba - SP, Brasil) a $50{ }^{\circ} \mathrm{C}$ e reservado para posteriores análises e caracterizações.

Para o estudo da relação entre três parâmetros e a variável resposta, no caso das nanopartículas a variável resposta é o tamanho, foi utilizada a metodologia de Box-Behnken. As variáveis independentes para esse estudo foram a temperatura $\left(5\right.$ a $\left.30^{\circ} \mathrm{C}\right)$, tempo de reação (10 a 60 minutos) e proporção ( 1 a 5 ) entre as soluções titulantes e titulado. Foram realizadas três repetições no ponto central, totalizando 15 ensaios. Os modelos de superfície obtidos a partir das respostas dos tratamentos foram ajustados por análise de variância (ANOVA), ao nível de $10 \%$ de probabilidade, utilizando SPSS Statistics software (Version 7.0, SPSS Inc. Chicago, IL).

\section{RESULTADOS E DISCUSSÃO}

A síntese de materiais inorgânicos em escala nano e micro com morfologias controláveis e bem definidas tem recebido considerável atenção. O motivo para o aumento da 
visibilidade desses materiais é que as suas propriedades têm uma grande correlação com fatores geométricos, como morfologia, dimensionalidade e o tamanho. Quando os tamanhos cristalinos de partículas são reduzidos ao intervalo de submicrômetros as partículas passam a possuir características como: proeminentes propriedades mecânicas/ópticas, alta área superficial específica e alta atividade química (DEVAMANI et al., 2016). Assim, o controle do tamanho de nanopartículas de carbonato de cálcio é crucial para otimizar sua performance (BABOU-KAMMOE et al., 2011). Na caracterização das nanoestruturas obtidas, a variável otimizada foi o tamanho, sendo as melhores condições de síntese aquelas que propiciaram menor tamanho de partícula.

As nanoestruturas de carbonato de cálcio produzidas a 50 e $25{ }^{\circ} \mathrm{C}$ apresentaram tamanhos de $167 \mathrm{~nm}$ e $224,2 \mathrm{~nm}$, respectivamente. Com base nesses resultados, constatou-se que o carbonato de cálcio ao ser submetido a uma temperatura maior apresentou uma nanoestrutura de tamanho menor em comparação ao carbonato de cálcio exposto a temperatura menor. Entretanto, a utilização de altas temperaturas como a de $50{ }^{\circ} \mathrm{C}$ não é interessante do ponto de vista industrial, visto que ocasiona aumento dos custos com energia e pode adicionar riscos de segurança ao processo.

Com o objetivo de avaliar a inter-relação entre outros parâmetros, além da temperatura, utilizou-se uma metodologia de superfície de resposta - Planejamento Box-Behnken. Este planeamento experimental é uma coleção de técnicas estatísticas para construir e explorar uma relação aproximada entre uma função objetiva e um conjunto de variáveis independentes (FAKHERI et al., 2014). A Tabela 1 abaixo apresenta os resultados dessa metodologia.

Tabela 1- Matriz do planejamento experimental e resultado experimental para o estudo da produção de nanoestrutura. Valores de tamanho $(\mathrm{nm})$ analisados no equipamento Nanozeta Sizer Malvern (Inglaterra, Reino Unido).

\begin{tabular}{|c|c|c|c|c|}
\hline Tratamento & $\begin{array}{c}\text { Temperatura } \\
\left({ }^{\circ} \mathrm{C}\right)\end{array}$ & Proporção & Tempo (min) & Tamanho (nm) \\
\hline \hline 1 & $5(-1)$ & $1(-1)$ & $35(0)$ & $249,5 \pm 11,6$ \\
\hline 2 & $30(1)$ & $1(-1)$ & $35(0)$ & $532,8 \pm 169,5$ \\
\hline 3 & $5(-1)$ & $5(1)$ & $35(0)$ & $217,6 \pm 52,1$ \\
\hline 4 & $30(1)$ & $5(1)$ & $35(0)$ & $670,9 \pm 117,8$ \\
\hline 5 & $5(-1)$ & $3(0)$ & $10(-1)$ & $426,6 \pm 80,2$ \\
\hline 6 & $30(1)$ & $3(0)$ & $10(-1)$ & $17,9 \pm 6,9$ \\
\hline 7 & $5(-1)$ & $3(0)$ & $60(1)$ & $185,7 \pm 16,2$ \\
\hline 8 & $30(1)$ & $3(0)$ & $60(1)$ & $119,7 \pm 68,6$ \\
\hline 9 & $17,5(0)$ & $1(-1)$ & $10(-1)$ & $664,5 \pm 151,5$ \\
\hline 10 & $17,5(0)$ & $5(1)$ & $10(-1)$ & $299,5 \pm 66,3$ \\
\hline 11 & $17,5(0)$ & $1(-1)$ & $60(1)$ & $28,5 \pm 5,8$ \\
\hline 12 & $17,5(0)$ & $5(1)$ & $60(1)$ & $32,9 \pm 7,0$ \\
\hline 13 & $17,5(0)$ & $3(0)$ & $35(0)$ & $248,5 \pm 11,6$ \\
\hline 14 & $17,5(0)$ & $3(0)$ & $35(0)$ & $284,4 \pm 94,4$ \\
\hline 15 & $17,5(0)$ & $3(0)$ & $35(0)$ & $269,1 \pm 64,8$ \\
\hline
\end{tabular}

A partir dos dados contidos na Tabela 1 construíram-se os gráficos de superfície 3D para avaliar as regiões de melhor resposta para a menor nanopartícula. As superfícies obtidas podem ser visualizadas nas Figura 1, Figura 2 e Figura 3. 
Figura 1- Superfície de resposta 3D identificando a influência da variação de temperatura e proporção para tempo fixo de 35 minutos.

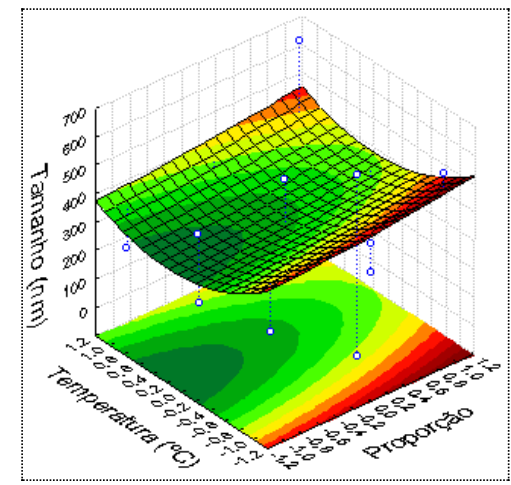

Figura 2- Superfície de resposta 3D identificando a influência da variação de temperatura e tempo para proporção fixa em 3.

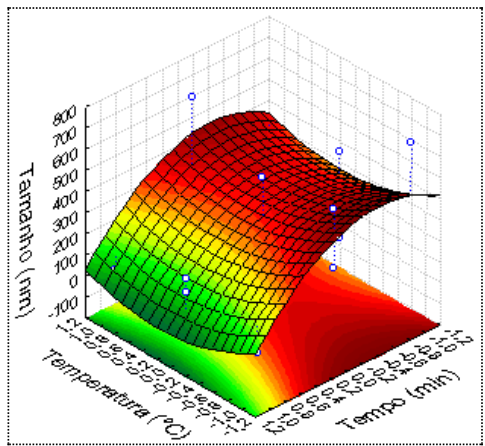

Figura 3- Superfície de resposta 3D identificando a influência da variação de proporção e tempo para temperatura fixa em $17,5^{\circ} \mathrm{C}$.

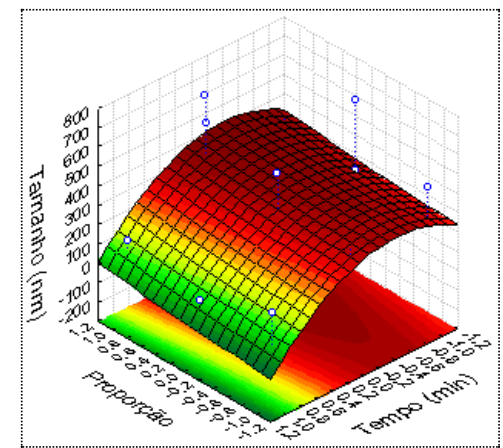

A região de interesse possui coloração verde escuro, uma vez que corresponde aos menores tamanhos de nanoestruturas. Através da superfície plotada, observa-se que quanto maior o tempo de gotejamento, ou seja, menor for a quantidade de líquido gotejada por tempo, menores são os tamanhos encontrados para as nanoestruturas. A diminuição do tamanho das nanoestruturas em um maior tempo de mistura pode ser devida ao efeito antagonista do amadurecimento de Ostwald, onde o tamanho médio da partícula começa a aumentar com o tempo de envelhecimento através da dissolução de pequenas partículas e crescimento de grandes partículas (BABOU- KAMMOE et al., 2012). Além disso, verifica-se 
que a proporção não possui influência significativa na variável resposta e que a temperatura ideal é em torno de $17,5^{\circ} \mathrm{C}$. A partir das análises feitas no software SPSS Statistics, das condições utilizadas no experimento e considerando possíveis custos energéticos, as melhores condições de síntese avaliadas para produção da nanoestrutura foram de $17,5^{\circ} \mathrm{C}$, em uma proporção de 1:1 por 60 min.

Com os dados obtidos foi gerada a ANOVA, um procedimento utilizado para comparar três ou mais tratamentos. $\mathrm{Na}$ análise de variância, vários grupos, também chamados de tratamentos, são comparados ao mesmo tempo (ALMEIDA et al.; 2012). A Tabela 2 abaixo apresenta os dados obtidos a partir da ANOVA.

Tabela 2- ANOVA dos dados obtidos a partir do software SPSS Statistics.

\begin{tabular}{|c|c|c|c|c|c|}
\hline Fator & SS & df & MS & F* $^{*}$ & P $^{*}$ \\
\hline \hline Proporção (L) & 49850,1 & 1 & 49850,1 & 2,9201 & 0,22961 \\
\hline Proporção (Q) & 203,1 & 1 & 203,1 & 0,0119 & 0,92311 \\
\hline Temperatura (L) & 1097,7 & 1 & 1097,7 & 0,0643 & 0,82351 \\
\hline Temperatura (Q) & 21486,3 & 1 & 21486,3 & 1,2586 & 0,37851 \\
\hline Tempo (L) & 69710,9 & 1 & 69710,9 & 4,0835 & 0,18071 \\
\hline Tempo (Q) & 87500,0 & 1 & 87500,0 & 5,1256 & 1,15187 \\
\hline Proporção (L) - Temperatura (L) & 7225,0 & 1 & 7225,0 & 0,4232 & 0,58208 \\
\hline Proporção (L) - Temperatura (Q) & 183406,0 & 1 & 183406,0 & 10,7436 & 0,08182 \\
\hline Proporção (Q) - Temperatura (L) & 27234,3 & 1 & 27234,3 & 1,5953 & 0,33387 \\
\hline Proporção (L) - Tempo (L) & 29360,8 & 1 & 29360,8 & 1,7199 & 0,32004 \\
\hline Proporção (Q) - Tempo (L) & 72860,8 & 1 & 72860,8 & 4,2681 & 0,17482 \\
\hline Temperatura (L) - Tempo (L) & 34108,5 & 1 & 34108,5 & 1,9980 & 0,29307 \\
\hline Erro & 34142,4 & 2 & 17071,2 & & \\
\hline Total SS & 657607,1 & 14 & & & \\
\hline
\end{tabular}

*Significativo ao nível de $10 \%$ de probabilidade.

L= Linear, Q= Quadrática.

$\mathrm{O}$ teste $\mathrm{F}$ apresenta a relação entre $\mathrm{o} \mathrm{F}_{\text {Calculado }} \mathrm{o} \mathrm{F}_{\text {Tabelado }}$. Quando a razão for maior que 1, a regressão é estatisticamente significativa, com relação entre as variáveis independentes e dependentes. Para o conjunto de dados, o valor de $\mathrm{F}_{\text {Tabelado }}$ foi de 2,4344. Para a proporção em regressão linear e temperatura em regressão quadrática, nota-se que o valor de $\mathrm{F}_{\text {Calculado }}$ é maior que do $\mathrm{F}_{\text {Tabelado }}(10,7436>2,4344)$, sendo a razão de $\mathrm{F}_{\text {Calculado }}$ e $\mathrm{F}_{\text {Tabelado }}$ maior que 1 e a regressão estatisticamente significativa. Outra maneira de avaliar a significância estatística de F é utilizando o p-valor. Como o p-valor é inferior ao valor de $\alpha(0,10)$, há o indicativo da relação entre as variáveis proporção, em uma regressão linear, e a temperatura em uma regressão quadrática.

\section{CONCLUSÃO}

Nanoestruturas de carbonato de cálcio foram obtidas sendo as melhores condições de síntese $17,5^{\circ} \mathrm{C}$, em uma proporção de $1: 1$ por $60 \mathrm{~min}$. Essas condições proporcionam um tamanho de partícula desejável e com menor gasto energético. Além disso, verificou-se que a proporção não tem influência significativa no tamanho de partícula e que quanto menor o tempo de gotejamento, menor o tempo de partícula. O teste ANOVA, ao nível de significância de $10 \%$ de probabilidade, permitiu inferir que a relação entre as variáveis proporção, é em uma regressão linear, e a temperatura em uma regressão quadrática. 


\section{AGRADECIMENTOS}

Os autores agradecem à FAPEMIG e CNPq pelo suporte financeiro concedido.

\section{REFERÊNCIAS}

ALMEIDA, E. C. O. de; MESQUITA, V. F. de M.; ALMEIDA, E. C. A. D. de.; RUSSO, S. L. Análise do tempo de atraso de pousos e decolagens no Aeroporto de Santa Maria em Aracaju . Rev. GEINTEC, v. 2, n.3, p. 285-296, 2012.

ASSIS, L. M de; ZAVAREZE, E. da R.; PRENTICE-HERNÁNDEZ, C.; SOUZA-SOARES, L. A. de. Review: characteristics of nanoparticles and their potential applications in foods. Braz. J. Food Technol., v. 15, n. 2, p. 99-109, 2012.

BABOU-KAMMOE, R.; HAMOUDI, S.; FAIÇAL, L.; BELKACEMI, K. Synthesis of $\mathrm{CaCO} 3$ nanoparticles by controlled precipitation of saturated carbonate and calcium nitrate aqueous solutions. Can. J. Chem. Eng., v. 90, n. 1, p. 26-33, 2012.

BOUWMEESTER, H; DEKKERS, S.; NOORDAM, M. Y.; HAGENS, W. I.; BULDER, A. S.; HEER, C. de; VOORDE, S. E. C. G. ten; WIJNHOVEN, H. J. P. M.; SIPS, A. J. A. M. Review of health safety aspects of nanotechnologies in food production. Regul. Toxicol. Pharmacol., v. 53, n. 1, p. 52-62, 2009.

DEVAMANI, R. H. P.; DEEPA, N.; GAYATHRI, J. Morphology and termal studies of calcium carbonate nanoparticles. J. Adv. Chem. Eng., v. 3, Issue 1, 2016.

FAKHERI, F.; ALAM, A. S.; MOHSENI, S.; KALHOR, R. R. Application of Box Behnken Experimental Design to Analysis Reaction Conversion of n-Butyl Acetate to n-Butanol. IJISET, v. 4, 2014.

HARUTA, S.; HANAFUSA, T.; FUKASE, H.; MIYAJIMA, H.; OKI, T. An effective absorption behavior of insulin for diabetic treatment following intranasal delivery using porous spherical calcium carbonate in monkeys and healthy human volunteers. Diabetes Technol Ther., v. 5, n. 1, p. 1-9, 2003.

JEONG, M. S.; PARK, J. S.; SONG, S. H.; JANG, S. B. Characterization of antibacterial nanoparticles from the scallop, Ptinopecten yessoensis. Biosci. Biotechnol. Biochem., v. 71, n. 9, p. 1247-1277, 2007.

MANJUSHA, H.; NEETHUMOL, V.; CHERIAN, A. B.; SREENIVASAN, P. V.; PAUL, J.; ANTONY, A. K. A. Synthesis and characterization of CaCO3 (Calcite) Nano Particles from cockle shells using chitosan as precursor. IJSER, v. 4, n. 10, 2014. 